\title{
Common Presentation of an Uncommon Small Intestinal Lymphoma: A Rare Case Entity
}

\author{
Sushma Bharti ${ }^{a}$ Jyotsna Naresh Bharti ${ }^{a}$ Mahendra Lodha ${ }^{b}$ \\ aDepartment of Pathology, All India Institute of Medical Sciences, Jodhpur, India; 'bepartment of General Surgery, \\ All India Institute of Medical Sciences, Jodhpur, India
}

\section{Keywords}

Follicular lymphoma · Grade 3b · Gastrointestinal lymphoma $\cdot$ Ileum $\cdot$ Immunohistochemistry

\begin{abstract}
Primary gastrointestinal NHL accounts for $30-40 \%$ of all extranodal NHL. Primary gastrointestinal lymphomas constitute $5 \%$ of total gut neoplasms. Bowel perforation is a severe life-threatening complication and sometimes initial presentation of gastrointestinal lymphoma. A 60-year-old man presented with complaints of abdominal pain, distension, nausea and vomiting. There was clinical suspicion of acute intestinal perforation, which was confirmed by radiology. The patient underwent emergency laparotomy. The resected bowel on histopathological examination and immunohistochemistry was diagnosed as the high-grade transformation of follicular lymphoma (FL). The patient received 6 cycles of chemotherapy and is doing well at 3 years of follow-up. Herein, we report this rare malignancy of the small intestine. FL mostly presents as a nodal disease but also involves the extranodal sites. The most common site of primary gastrointestinal-follicular lymphoma (GI-FL) is the small intestine. The cellular and molecular characteristic of GI-FL is different from that of the nodal FL. Extranodal FL is usually localized, but the prognosis of transformed FL is low, and these are
\end{abstract}

karger@karger.com www.karger.com/gat

Karger!"

BOPEN ACCESS
(C) 2021 The Author(s)

Published by S. Karger AG, Basel

This article is licensed under the Creative Commons AttributionNonCommercial-NoDerivatives 4.0 International License (CC BYNC-ND) (http://www.karger.com/Services/OpenAccessLicense) Usage and distribution for commercial purposes as well as any distribution of modified material requires written permission. managed by surgery followed by chemotherapy. High clinical suspicion and extensive sampling of perforated bowel are essential to diagnose the high-grade transformation of FL.

C 2021 The Author(s).

Published by S. Karger AG, Basel

\section{Background}

Primary gastrointestinal NHL accounts for $30-40 \%$ of all extranodal NHL [1]. Primary GI lymphomas constitute $5 \%$ of total gut neoplasms [2]. Follicular lymphoma (FL) of the GI tract is rare and comprises $4 \%$ of primary GI lymphomas. Bowel perforation and peritonitis are severe life-threatening complications and sometimes initial presentation of gastrointestinal lymphoma. Herein, we report the first case of high-grade transformation of ileum FL to the best of our knowledge after an extensive search in PubMed.

\section{Case Report}

A 60-year-old man presented with complaints of severe abdominal pain. On examination, the bowel sound was absent and rebound tenderness was present. Clinically and radiologically, in- 


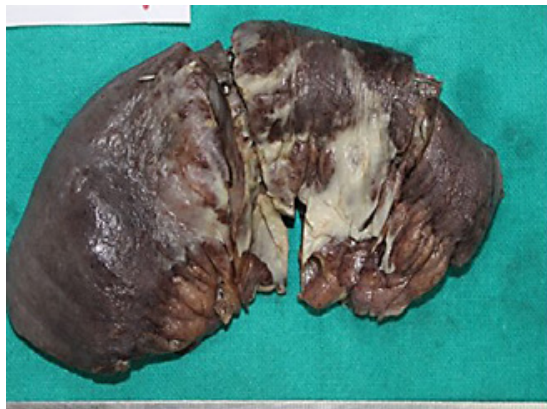

a

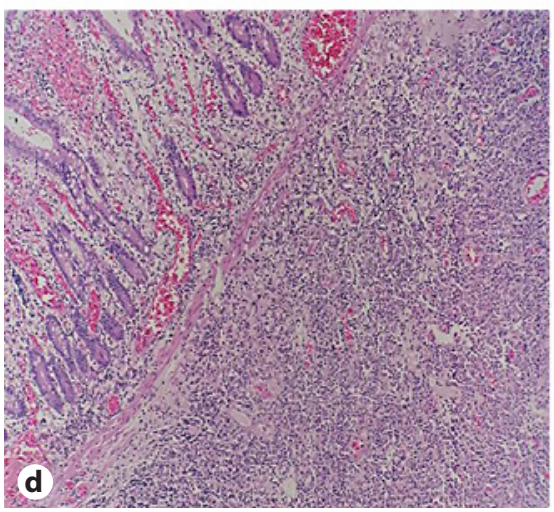

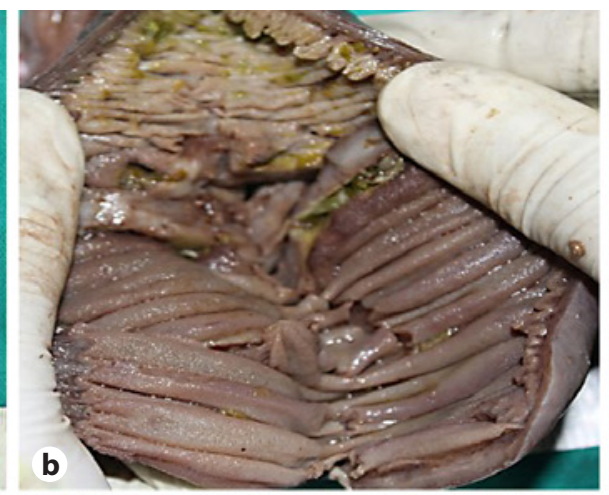
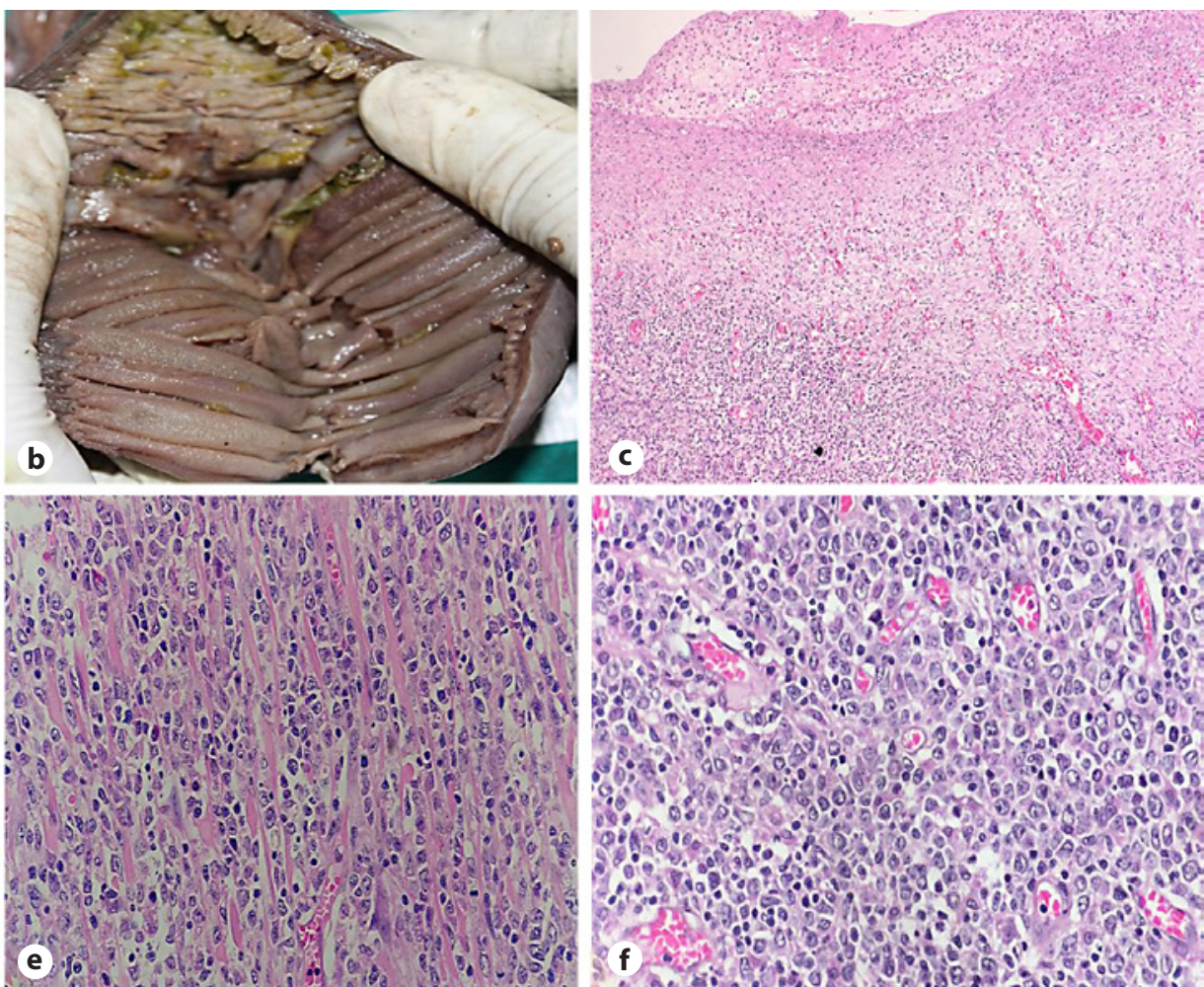

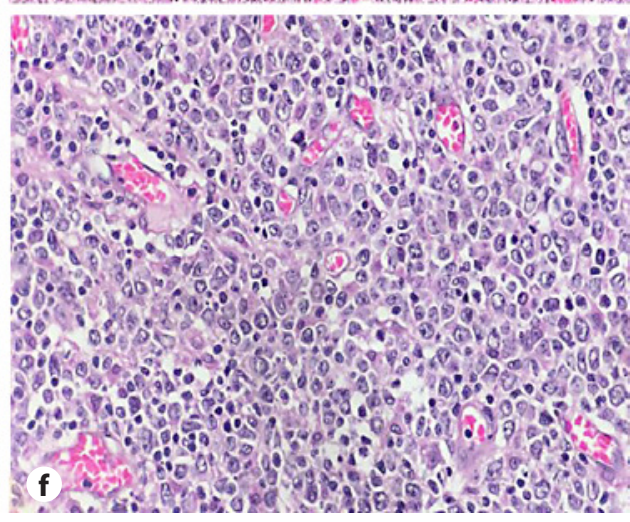

Fig. 1. a Resected ileum with exudate covered serosa. b Ileum showing perforation of the mucosal surface. c Granulation tissues and serosal exudate at the perforation site. (H\&E, $\times 200)$. d Tumor cells infiltrating lamina propria and the submucosa $(\mathrm{H} \& \mathrm{E}, \times 100)$. e Tumor cells infiltrating into the muscularis propria $(\mathrm{H} \& \mathrm{E}, \times 400)$. f Atypical lymphoid cells $(\mathrm{H} \& \mathrm{E}, \times 400)$.

testinal perforation was considered. Emergency laparotomy and bowel resection were done and sent for histopathological examination. We received resected thickened wall ileum with perforation, which was covered with exudate over the serosal surface (Fig. 1a, b). Sections from the perforation sites showed ulcerated mucosa with underlying granulation tissue and serosal exudate (Fig. 1c). Transmural diffuse sheets of atypical lymphocytes expanding the unremarkable mucosa were also seen (Fig. 1d). The tumor cells were large, non-cleaved (centroblasts) with 1-2 nucleoli abutting the nuclear membrane, moderate to scant cytoplasm, with indistinct cell border along with few small cleaved cell type (Fig. 1e, f). The follicle formation was $<10 \%$. On immunohistochemistry, the cells were positive for LCA, CD79a, CD20, and CD10, Bcl2, and Bcl6 and negative for CK, CD117, CD3, CD5, Cyclin D1, and MUM-1. The Ki-67 was $>85 \%$ (Fig. 2a-f). The features were of a high-grade transformation of FL (FL Grade $3 b$ with DLBCL component). We do not have FISH or a PCR setup in our department for further confirmation. However, the ancillary technique is not mandatory according to the essential and desirable criteria by the WHO for diagnosis.

The patient had no B-symptoms. The blood and marrow were unremarkable with no lymphadenopathy and organomegaly. Hence, this was considered as primary gastrointestinal-follicular lymphoma (GI-FL) instead of nodal FL dissemination, although primary GI-FL is quite rare. Nodal FL usually affects the blood and bone marrow. However, in extranodal FL, like primary GI-FL, it does not affect the blood or bone marrow.

The principal differential diagnosis of GI-FL is reactive follicular hyperplasia, in which follicles are not tightly packed and are of variable size, shape, well-defined mantle, and variable size population of cells with many tingible body macrophages. On immunohistochemistry, the reactive follicle is BCL2 negative. Extranodal MALT lymphoma shows diffuse to vague nodular infiltrates of small- to medium-sized cells with pale cytoplasm. The cells are immunoreactive to CD20, and negative to CD10 and Bcl6. The mantle cell lymphoma shows diffuse to vaguely nodular infiltrate of small- to medium-sized cells with pale cytoplasm. The cells are immunoreactive to CD20, CD5, and cyclin D1, and negative to CD10. In DLBCL, cells may appear centroblastic or immunoblastic and are immunoreactive to CD10, BCL2, BCL6, and MUM-1, depending upon molecular subtypes. The CLL/SLL shows smallsized cells with inconspicuous nucleoli and scant cytoplasm. The cells are immunoreactive to CD5 and CD 23. The patient received 6 cycles of R-CHOP (rituximab monotherapy-cyclophosphamide, doxorubicin, vincristine, and prednisone). PETCT did not reveal any evidence of metabolic activity, and the patient is doing well at the end of 3 years.
Bharti/Bharti/Lodha 

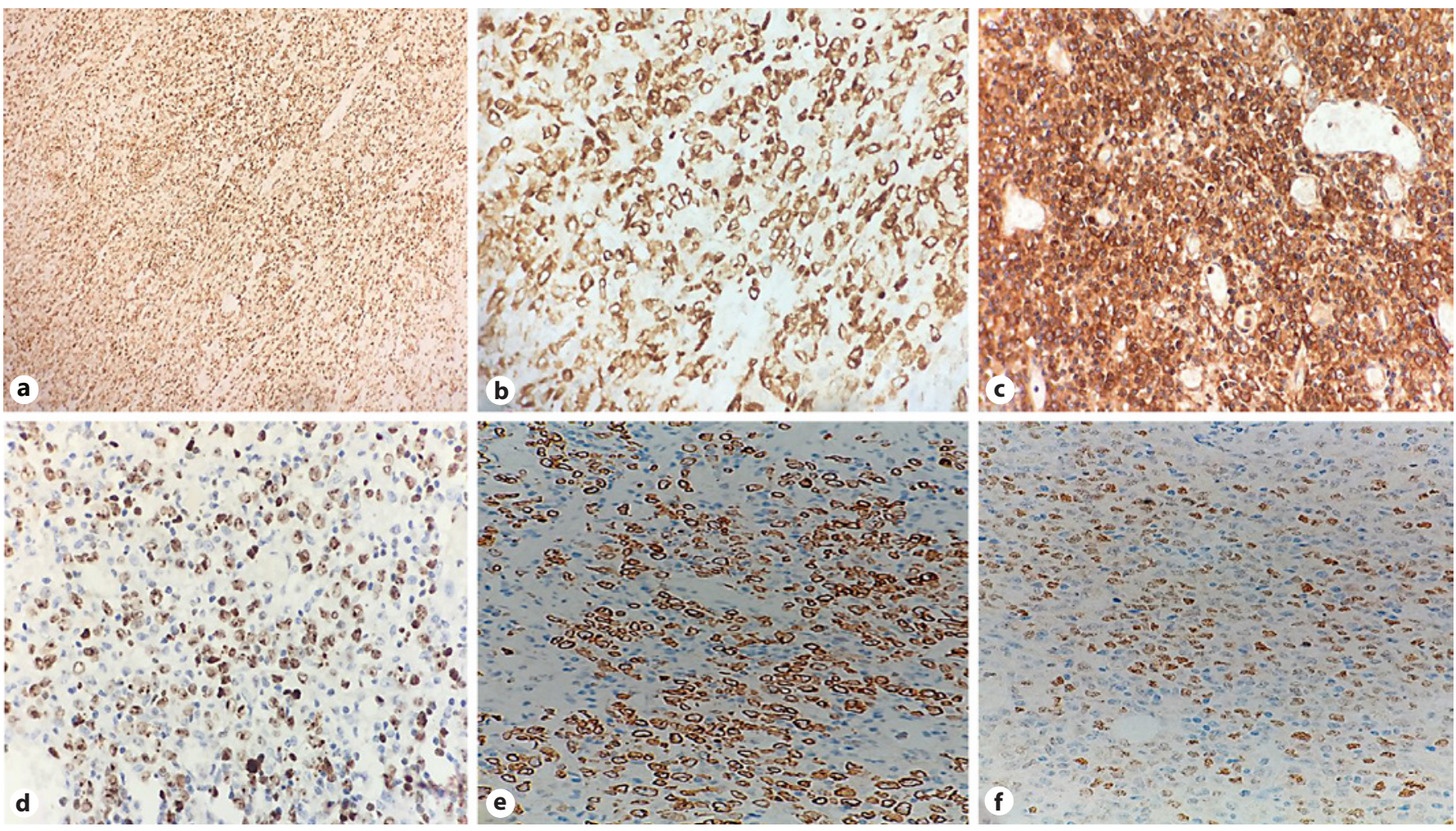

Fig. 2. a LCA immunoreactivity (IHC, $\times 100)$. b CD79a immunoreactivity (IHC, $\times 200)$. c CD10 immunoreactivity $(\mathrm{IHC}, \times 200)$. d Ki-67 > 85\% (IHC, $\times 100)$. e Bcl2 immunoreactivity (IHC, $\times 100)$. f Bcl6 immunoreactivity $(\mathrm{IHC}, \times 100)$.

\section{Discussion}

Lymphoma can be nodal and extranodal. The most typical site of extranodal lymphoma is GIT $[3,4]$. These constitute approximately $10-15 \%$ of all NHL, commonly affecting the stomach, small intestine (SI), and large intestine (LI) [1]. A lymphoid neoplasm can be mature B, $\mathrm{T}$, and less commonly extranodal NK/T cell types. The commonest B-cell GIT lymphomas are MALT lymphoma and DLBCL. A less common one is Burkitt lymphoma, FL, MCL, and anaplastic large-cell lymphoma ALKpositive and lymphomatoid granulomatosis.

FL is also known as follicle (germinal) center cell lymphoma and centroblastic/centrocytic lymphoma. It is an indolent B-NHL. Mostly present as nodal, but common extranodal sites are skin, testis, orbit, thyroid, salivary gland, and GIT [5]. The primary location of GI-FL is the second part of the duodenum [2] but can be present in other parts of SI [6]. The median age is $44-71$ years, with almost equal sex predilection. Extranodal FL is usually localized and presents as abdominal pain, intussuscep- tion, bleeding, and sometimes incidentally. On endoscopy and gross, it appears as multiple nodular lesions (1-2 $\mathrm{mm}$ ), mucosal ulceration, perforation, hyperplasia, polyps, infiltrative lesions, and sometimes as thickening of the wall. High-grade lymphoma like DLBCL usually presents as ulcers, perforation, and multiple stenoses [3, 5, 7]. Similarly, in the present case, the patient presented with perforation peritonitis.

Extranodal FL has similar morphology as nodal FL [6], with tight-packed neoplastic follicles effacing the nodular architecture and absence of tangible body macrophages. Follicles comprised a monomorphic population of centrocytes and centroblasts. Cells are positive for B-cell antigen, $\mathrm{CD} 10$, and $\mathrm{Bcl} 2$. $\mathrm{CD} 10$ is positive in approximately $60 \%$ cases and absent in Grade 3B FL, but the BCL6 is retained. GI-FL does not show follicular dendritic cell expression [5]. MUM-1/IRF4 is negative in low-grade FL and positive in Grade 3 FL. BCL6 is downregulated, and Bcl2 is positive in $85-90 \%$ of grade I/II FL and $50 \%$ of Grade 3 FL. Neoplastic follicles expand the mucosa without destroying the mucosa. The tumor cells are Bcl2 pos- 
itive, which is essential for the diagnosis of FL. Akiyama et al. [8] found sheets of large centroblasts positive for CD20, CD10, BCL6, BCL2, and negative for CD5, MUM1. The MIB-1 is high. These findings were consistent with the diagnosis of DLBCL transformed from duodenal FL, similar to the present study, in which atypical cells were positive for CD20, CD10, BCL6, BCL2, and negative for CD5 and MUM-1 and had high MIB-1 index. However, cellular and molecular characterization of GI-FL is different from nodal FL and is similar to MALT lymphoma [9].

By definition, histological change from FL grade I/II to DLBCL or Burkitt/Burkitt-like morphology or Grade 3b FL is called when diffuse sheets of large centroblasts present, representing DLBCL. Approximately $5 \%$ of FL is Grade 3 and seen at about 3\% per year for the first 10 years of observation $[10,11]$. Also, the prognosis of transformed FL is dismal and treated with a surgery followed by chemotherapy. However, no current guidelines have been approved for the management of low- and highgrade FL.

To date, only a few cases have been reported in the literature, and all reported cases are of low grade and involving the intestine multifocally or unifocally. The first case of histological transformation was published by Takata et al. [7], who reported primary duodenal FL showing histological transformation to DLBCL. Takata et al. [5] and Tanigawa et al. [12] also found that only a few cases of histological transformation of GI-FL reported till 2019, and all are of duodenal FL transformation. Nishimura et al. [3] reported a case of transformation of jejunoileal FL into DLBCL detected using double-balloon enteroscopy. Grade 3b FL is a distinct entity, which has additional DLBCL components. Pure FL Grade $3 b$ is a rare entity. DLBCL associated FL Grade $3 \mathrm{~b}$ component has a translocation involving Bcl6, and a high ki-67 index is considered as one of the hallmarks of DLBCL associated FL Grade 3 [13]. However, there are no definitive criteria defined, as there are very few reported cases in the literature. Bautista-Quach et al. [1] and Jaffe et al. [14] found some genetic features overlap, however, with rearrangements of BCL6 seen in 6-14\% of FLs and of BCL2 in 20$30 \%$ of DLBCLs.

\section{Conclusion}

Intestinal perforation causes can be multiple, from infective to carcinoma and lymphoma. These are often missed or misdiagnosed because of incomplete or inadequate sampling from erosion, ulcerated, and perforated areas showing small atypical cells. Therefore, high clinical suspicion, representative biopsy, and extensive evaluation of surgically resected specimens are mandatory for correct diagnosis.

\section{Acknowledgements}

We would like to thank all those who helped in writing this article.

\section{Statement of Ethics}

The patient gave written informed consent for the publication of data and images.

\section{Conflict of Interest Statement}

There are no conflicts of interest regarding the publication of this article.

\section{Funding Sources}

The authors did not receive any funding.

\section{Author Contributions}

Dr. Sushma Bharti, Senior Resident: manuscript writing and searching the references. Dr. Jyotsna Naresh Bharti, Associate Professor: diagnosis, manuscript editing, and provided the gross and microscopy images. Dr. Mahendra Lodha, Associate Professor: Operated the case and provided the clinical details.

\section{References}

1 Bautista-Quach MA, Ake CD, Chen M, Wang J. Gastrointestinal lymphomas: morphology, immunophenotype, and molecular features. J Gastrointest Oncol. 2012;3(3):209-25.

2 Chouhan J, Batra S, Gupta R, Guha S. Gastrointestinal follicular lymphoma: using the primary site as a predictor of survival. Cancer Med. 2016;5:2669-77.

3 Nishimura T, Kuwai T, Imagawa H, Kohno H. Transformation of jejunoileal follicular lymphoma into diffuse large B-cell lymphoma detected using double-balloon enteroscopy. BMJ Case Rep. 2018 Jul 18;2018: bcr2018224467.

4 Gou HF, Zang J, Jiang M, Yang Y, Cao D, Chen XC. Clinical prognostic analysis of 116 patients with primary intestinal non-Hodgkin lymphoma. Med Oncol. 2012;29(1):22734.
Bharti/Bharti/Lodha

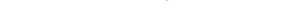


5 Takata K, Miyata-Takata T, Sato Y, Iwamuro M, Okada H, Tari A, et al. Gastrointestinal follicular lymphoma: current knowledge and future challenges. Pathol Int. 2018;68(1):1-6.

6 Takata K, Okada H, Ohmiya N, Nakamura S, Kitadai Y, Tari A, et al. Primary gastrointestinal follicular lymphoma involving the duodenal second portion is a distinct entity: a multicenter, retrospective analysis in Japan. Cancer Sci. 2011;102(8):1532-6.

7 Miyata-Takata T, Takata K, Sato Y, Taniguchi $\mathrm{K}$, Takahashi Y, Ohara N, et al. A case of diffuse large B-cell lymphoma transformed from primary duodenal follicular lymphoma. Pathol Int. 2014;64(10):527-32.
8 Akiyama S, Izutsu K, Ota Y, Imamura T, Ogawa $\mathrm{O}$, Wake A, et al. A case report of the histologic transformation of primary follicular lymphoma of the duodenum. Medicine. 2014; 93(26):e165.

9 Takata K, Tanino M, Ennishi D, Tari A, Sato Y, Okada H, et al. Duodenal follicular lymphoma: comprehensive gene expression analysis with insights into pathogenesis. Cancer Sci. 2014;105(5):608-15.

10 Wong E, Dickinson M. Transformation in follicular lymphoma: biology, prognosis, and therapeutic options. Curr Oncol Rep. 2012; 14(5):424-32.

11 Montoto S, Fitzgibbon J. Transformation of indolent B-cell lymphomas. J Clin Oncol. 2011;29(14):1827-34.
12 Tanigawa T, Abe R, Kato J, Hosoe N, Ogata H, Kameyama K, et al. Histological transformation in duodenal-type follicular lymphoma: a case report and review of the literature. Oncotarget. 2019;10(36):3424-9.

13 Katzenberger T, Ott G, Klein T, Kalla J, Müller-Hermelink HK, Ott MM. Cytogenetic alterations affecting BCL6 are predominantly found in follicular lymphomas grade $3 \mathrm{~B}$ with a diffuse large B-cell component. Am J Pathol. 2004;165(2):481-90.

14 Jaffe ES, Harris NL, Stein H, Vardiman JW, editors. Pathology and Genetics of Tumours of Hematopoietic and Lymphoid Tissues. Lyon: IARC Press; 2001. 